\title{
An untethered free-fall vertical profiler for deep ocean mixing measurements
}

\author{
Xiaoyang Xu \\ Engineering college \\ Ocean University of China \\ Qingdao, China \\ Shengzong Zhang \\ Institute of Deep-sea Science and Engineering, \\ Chinese Academy of Sciences \\ Sanya, China \\ Dazhen $\mathrm{Xu}$ \\ Institute of Deep-sea Science and Engineering, \\ Chinese Academy of Sciences \\ Sanya, China \\ Shiliu Peng \\ Institute of Mechanics, Chinese Academy of
}

Abstract: The research on the process of deepocean turbulent mixing has become one of the frontier technologies in the field of scientific research. Currently, the most commonly used profiler is the tethered vertical profiler, however its measuring depth is limited by the length of the rope. An untethered free-fall vertical profiler which can profile down to a depth of $\mathbf{4 0 0 0} \mathrm{m}$ is developed by Institute of Deep-sea Science and Engineering, Chinese Academy of Science. The profiler equipped with microstructure sensors (two orthogonal shear probes and one fast response thermistor) is used to measure the ocean turbulent mixing and estimate the rate of dissipation of turbulent kinetic energy (TKE) and temperature gradient. The sea trail of profiler was conducted in the South China Sea and the shear spectra from 920 to $1020 \mathrm{~m}$ agree with the Nasmyth spectrum.

Keywords-untethered vertical profiler, microstructure sensors, turbulence, the rate of dissipation of TKE
Sciences

Beijing, China

Jian Liu

Institute of Deep-sea Science and Engineering, University of Chinese Academy of Sciences Sanya, China

Xueqian Yang

Institute of Deep-sea Science and Engineering, Chinese Academy of Sciences

Sanya, China

Chuan Tian*

Institute of Deep-sea Science and Engineering, Chinese Academy of Sciences

Sanya, China

\section{INTRODUCTION}

Ocean turbulent mixing plays a crucial role in ocean thermohaline dissipation and energy circulation. It is also important for sediment transport, marine ecological environment, suspended solids distribution and climate ${ }^{[1]}$. Ocean energy moves from large to dissipative scales. Ocean microstructure (less than $1 \mathrm{~m}$ vertical) energy finally dissipates in the form of turbulent mixing ${ }^{[2]}$. As the scale of turbulence is usually less than $0.01 \mathrm{~m}$ and the measurements of turbulence are easily influenced by mechanical vibrations, instruments used to measure turbulence require high accuracy and low noise. The turbulence measurements require the improvement of sensors and platforms. In 1962, Grant et al used the hot-film anemometer to measure microstructure velocity fluctuations ${ }^{[3]}$. In 1972, T. Osborn measured microstructure velocity fluctuations using the shear probe which is not sensitive to temperature fluctuations ${ }^{[4]}$. 
Nowadays the shear probe has become the most suitable sensor to measure microstructure velocity fluctuations. The early turbulence measurements were performed with horizontal profilers, but by the late 1960s, most measurements were conducted with vertical profilers ${ }^{[5]}$. Vertical profilers are propelled by gravity and moves smoothly through the water, which make they are high-accuracy instruments. Ocean turbulent mixing researches are gradually developed to the deep ocean, but ocean mixing measurement instruments are rare, especially in the deep ocean. A number of vertical profilers must link to the ship with the tether, however, the length of the tether limits the profiler's descent depth. This paper introduces an untethered freefall vertical profiler which is developed by Institute of Deep-sea Science and Engineering, Chinese Academy of Sciences. The untethered free-fall vertical profiler measures the turbulent mixing in the deep ocean. It can descend close to the ocean bottom. Furthermore, the profiler avoids the disturbance from the hull vibration and minimizes the generation of eddies brought by the instrument motion.

The outline of this paper is as follows. The introduction of instrumentation is described in the second part. The sea trail in the South China Sea (SCS) at $18^{\circ} 11.213^{\prime} \mathrm{N}, 114^{\circ} 31.167^{\prime} \mathrm{E}$ is presented in the third part. The data processing and analysis are presented in the fourth part. The fifth part draws the conclusion.

\section{INSTRUMENTATION}

The untethered free-fall vertical profiler (Fig. 1) autonomously descends with a speed of 0.5-0.55 $\mathrm{m} / \mathrm{s}$, which is controlled by the relationship of their buoyancy and the gravity of the weight. The nose cabin of the profiler is mounted with microstructure sensors (two airfoil shear probes, one fast response thermistor). The electronic cabin is equipped with circuit boards which contain data acquisition unit, analog signal unit, control unit, power supply unit and data logging unit. The battery cabin supplies power for the electronic system though watertight cables. When the profiler freely falls close to the seafloor, it releases the weight and rises to the surface at a speed of $1.0 \mathrm{~m} / \mathrm{s}$. Then the profiler is recovered with the help of the Iridium beacon which provides location information.

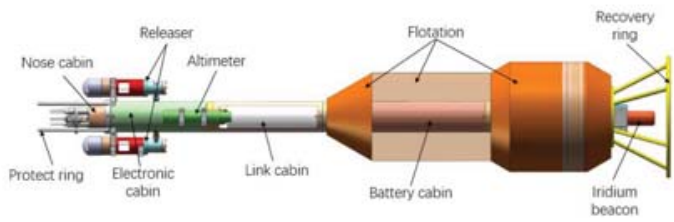

Fig. 1 The structure of the untethered free-fall vertical profiler

\section{a. The untethered free-fall vertical profiler}

The profiler is $2.3 \mathrm{~m}$ long and $0.4 \mathrm{~m}$ in diameter, and has a mass of $100 \mathrm{~kg}$ (in air) (Fig. 2). The microstructure sensors are parallelly mounted on the nose cabin of the profiler. We choose PNS06 airfoil shear probe to measure turbulence velocity fluctuations, and the airfoil connects with piezoceramic through a cantilever which can amplify mechanical signals. The piezo-ceramic transforms mechanical signals into analog signals. Two shear probes installed orthogonally, respond to two directions of vertical velocity fluctuations (W and U), respectively. FP07, a fast response thermistor whose response time is only $7 \mathrm{~ms}$, is used to measure turbulence temperature variances.

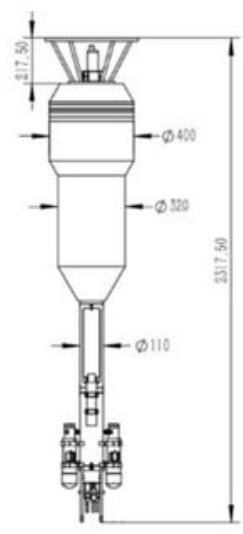

Fig.2 Engineering drawing ( $\mathrm{mm}$ ) of the untethered free-fall vertical 
A conductivity temperature and depth (CTD) sensor integrated within the nose cabin of the profile is used to acquire seawater salinity and density. Furthermore, a high accuracy attitude sensor is integrated into electronic cabin to evaluate profiler attitude in downcast.

The electronic cabin is equipped with circuit boards which contain data acquisition unit, analog signal unit (pre-amplifier, filtering, 16bit A/D converter), control unit, power supply unit and data logging unit. The sampling frequency of PNS06 and FP07 is set to $1024 \mathrm{~Hz}$. The MTI's sampling frequency is $200 \mathrm{~Hz}$. Electronic circuitry amplifies and filters the signals produced by microstructure sensors and records signals for later analysis. All data measured by sensors are saved in SD card. The main voltage is $12 \mathrm{~V}$. The voltage range of all analog signal units is from $-5 \mathrm{~V}$ to $+5 \mathrm{~V}$. The voltage of the control unit is $3.3 \mathrm{~V}$. The electronic cabin has five external connectors. One 4-pin underwater connector connects two 2-pin releasers through a crossover coupling. One 8-pin underwater connector connects the altimeter. One 8-pin underwater connector is used to transmit data to the host computer. One 4-pin and one 8-pin underwater connectors connect the battery cabin.

The battery cabin is surrounded by cylindrical floating body material and it is equipped with lithium batteries powering the electronic system and releasers through two watertight cables. The string around the end of the floating body can avoid the trailing vortex. The rear section of the profiler installs a titanium recovery ring and an Iridium beacon which is used to determine the position of the profiler.

\section{b. Release system}

The release system is equipped with two release mechanisms. The primary mechanism is the electromagnet adsorption release. When it fails to release the weight, there is another release mechanism, the dissolving ring release, to ensure that the profiler releases the weight and ascends to the surface. The release system contains an altimeter, a depth sensor and two releasers. The releasers use the hydraulic technology to balance the extremely high pressure in the deep ocean.

The electromagnet adsorption release is simple and effective. Electrify the electromagnet, then the iron core is adsorbed, the hook rotates and the weight slips out freely. A release signal is triggered upon: 1. When the distance from the seafloor measured by the altimeter is consistent with the set value; 2 . When the profiler descends to the designed depth; 3 . When the profiler in the ocean exceeds the selected duration.

The dissolving ring release depends on the corrosion of the dissolving ring which dissolves in seawater in $3.5-4 \mathrm{~h}$ at $4{ }^{\circ} \mathrm{C}$. The dissolving ring connects the weight with the hook. When reaching the maximum dissolving time, the dissolving ring dissolves. Then the weight is released (Fig. 3).

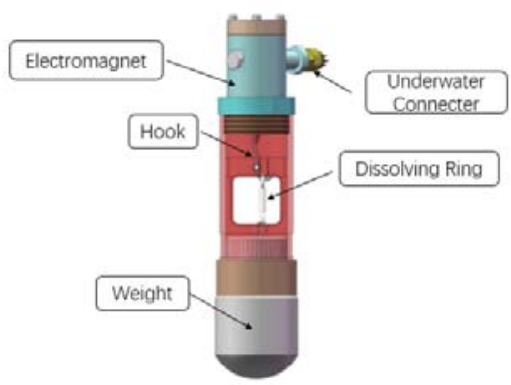

Fig. 3 The structure of Releaser

\section{SEA TRAIL}

The untethered free-fall vertical profiler was deployed in the South China Sea at $18^{\circ} 11.213^{\prime} \mathrm{N}$, $114^{\circ} 31.167^{\prime} \mathrm{E}$. The profiler fell freely at a speed of $0.52-0.58 \mathrm{~m} / \mathrm{s}$ and it profiled down to a depth of $1020 \mathrm{~m}$, which took about 32 minutes.

\section{RESULTS}

\section{a. Fall rate and attitude of the profiler}

For the sea trail in the South China Sea, the profiler descended at a speed of $0.58 \mathrm{~m} / \mathrm{s}$ near the surface and slowed to $0.52 \mathrm{~m} / \mathrm{s}$ at the bottom, 
which was due to the increasing density of seawater with depth (Fig. 4). The MTI attitude sensor records the profiler's 3-Direction acceleration signals and the profiler's tilts (pitch, roll, and head). The typical pitch angle was $\pm 1^{\circ}$, and the roll angle was $\pm 1.2^{\circ}$.

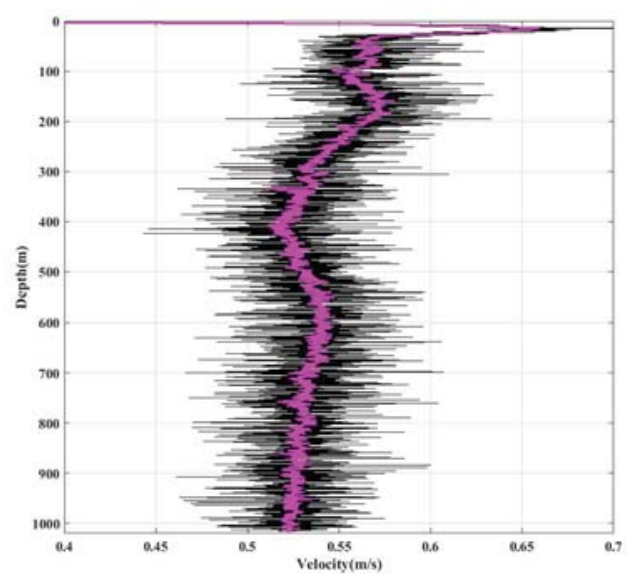

Fig. 4 The profiler fall rate (black) and after smooth fall rate (purple)

\section{b. The rate of dissipation}

The rate of dissipation of turbulent kinetic energy can be estimated by the data measured by the shear probe. The piezo-ceramic in the shear probe converts the turbulence velocity into the voltage signal, and the voltage signal is amplified by 11 times, then the output voltage signal can be used to calculate the rate of dissipation of turbulent kinetic energy. The output voltage produced by the shear probe is as follows ${ }^{[6]}$ :

$$
E_{p}=2 \sqrt{2} S u \omega
$$

Where $\mathrm{u}$ is the speed of the profiler relative to the water, $\omega$ is either one of the two horizontal components of velocity. $S$ is the sensitivity of the shear probe. According to Taylor frozen hypothesis (the time-series transformation to the spatial-domain):

$$
\frac{\partial}{\partial \mathrm{t}}=\mathrm{U} \frac{\partial}{\partial \mathrm{x}}
$$

We can calculate shear signal in directions of
$\mathrm{W}$ and $\mathrm{U}$, respectively: $\frac{\partial \omega}{\partial \mathrm{x}}=\frac{E_{S}}{2 \sqrt{2} S U^{2}}$, $\frac{\partial u}{\partial x}=\frac{E_{S}}{2 \sqrt{2} S W^{2}}$

If ocean turbulence is isotropic, the dissipation rate of TKE $\varepsilon$ can be estimated by the following equation ${ }^{[7]}$ :

$$
\varepsilon=\frac{15}{2} v \overline{\left(\frac{\partial w}{\partial z}\right)^{2}}=\frac{15}{2} v \int_{k_{\min }}^{k_{\max }} \Psi(k) d k
$$

where $v$ is the kinematic molecular viscosity, the over-line indicates a spatial or ensemble average, $w$ is either one of the two horizontal components of velocity, $\mathrm{z}$ is the vertical coordinate, $\Psi(k)$ is the wavenumber spectrum of the vertical shear, $\mathrm{k}$ is the vertical wavenumber in the unit of cpm (cycles per meter), and $k_{\min }$ to $k_{\max }$ is the wavenumber range of spectrum.

For the South China Sea, the dissipation rate of TKE is calculated with two shear probes. In Fig.6 (a) and (b) selected the depth range from 920 to $1020 \mathrm{~m}$, the red point is the dissipation rate before noise removal processing and the blue point is the dissipation rate after noise removal processing. In the depth of $937.52 \mathrm{~m}$, the dissipation rate of TKE of shear 1 (blue line) is $\varepsilon 1=6.7552 \times 10^{-10} \mathrm{~W} \mathrm{~kg}^{-1}$ and the dissipation rate of TKE of shear 2 (red line) is $\varepsilon 2=4.153 \times 10^{-10} \mathrm{~W} \mathrm{~kg}^{-1}$ (Fig. 6(c)). The dashed line is Nasmyth spectrum and the vertical dashed line is the cut-off wavenumber. Before the cut-off wavenumber, the observed shear spectra agree with the Nasmyth spectrum. In Fig.6 (d) is the dissipation rate of TKE in the depth of 1007.92 m. In Fig.6 (c) and (d), the dashed line is Nasmyth spectrum and the vertical dashed line is the cut-off wavenumber. Before the cut-off wavenumber, the observed shear spectra agree with the Nasmyth spectrum. 

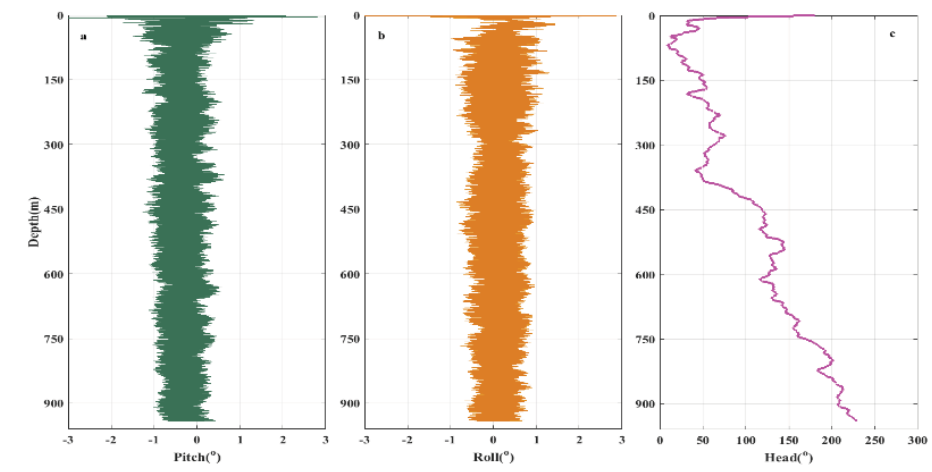

Fig. 5 MTI sensor collected data of pitch (green), roll (gold), and head (purple)
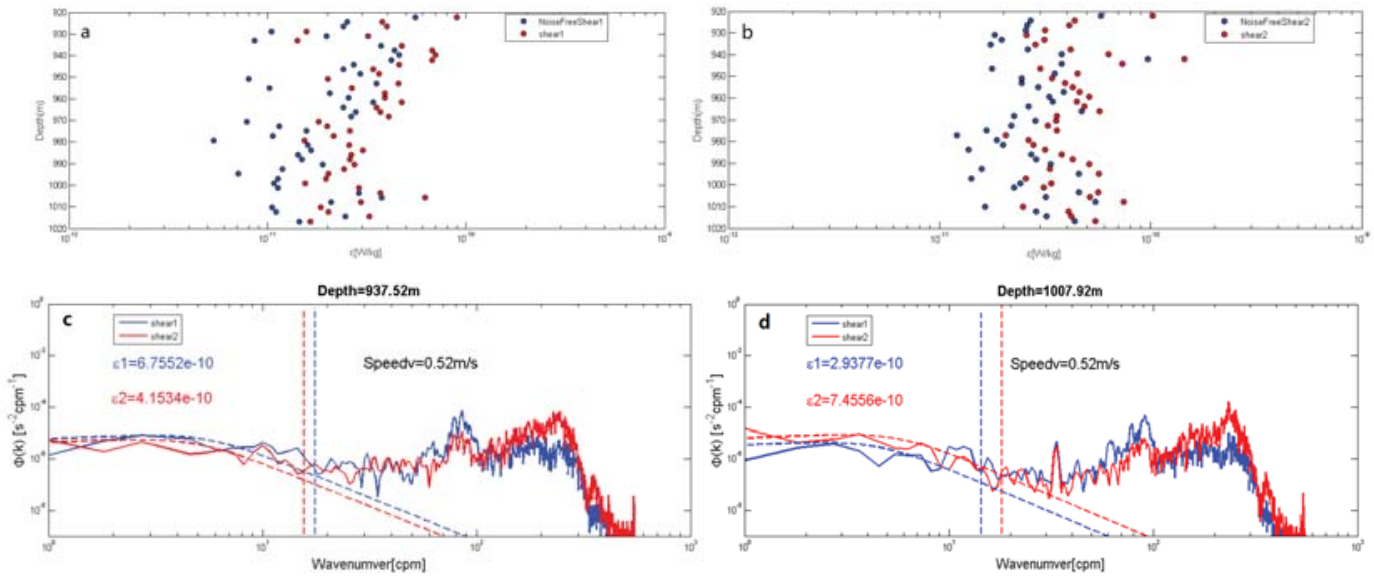

- $\quad$ Fig. 6 (a) The dissipation rate of TKE (b) The dissipation rate of TKE after noise removal processing.

(c) and (d) Comparison of the observed shear spectra and the Nasmyth spectrum (dashed line).

\section{CONCLUSION}

An untethered free-fall vertical profiler, developed by Institute of Deep-sea Science and Engineering, Chinese Academy of Science, has been deployed in the South China Sea to measure the dissipation rate of turbulent kinetic energy and temperature gradient. The profiler successfully descends to a depth of $1020 \mathrm{~m}$ and observes vertical turbulent mixing. The profiler's pitch angle was $\pm 1^{\circ}$ and the roll angle was $\pm 1.2^{\circ}$. The spectra of shear agree with the Nasmyth spectrum.

\section{ACKNOWLEDGMENT}

We thank the professor Shiliu Peng for his guidance. We also thank the Institute of Deep-sea Science and Engineering, Chinese Academy of Science for the platform of vessel "TAN SUO YI HAO". Finally, we appreciate the help of whole research team.

\section{REFERENCES}

[1] Munk W, Wunsch C. Abyssal recipes II: Energetics of tidal and wind mixing $[\mathrm{J}]$. Deep Sea Research Part I: Oceanographic Research Papers, 1998, 45(12): 1977-2010

[2] Munk, W. H. Internal waves and small-scale processes. Evolution of Physical Oceanography p: 264-291, 2010.

[3] Grant, H. L., R. W. Stewart, and A. Moilliet, 1962: Turbulence spectra from a tidal channel. Journal of Fluid Mechanics, 12, 241-263

[4] Osborn, T. R., 1974: Local vertical profiling of velocity microstructure. Journal of Physical Oceanography, 4, 109-115

[5] Grant, H. L., A. Moilliet, and W. M. Vogel, 1968a: Some observations of the occurrence of turbulence in and above the thermocline. Journal of Fluid Mechanics, 34, 443-498. 
[6] Levine, E. R., Lueck, R. G. "Turbulence measurement from an autonomous underwater vehicle". Journal of Atmospheric \& Oceanic Technology, vol. 16(11), pp. 1533-1544, 1999.

[7] Fer, I., Paskyabi, M. B. "Autonomous ocean turbulence measurements using shear probes on a moored instrument". Journal of Atmospheric \& Oceanic Technology, vol. 31(2), pp. 474-490, 2013. 